\title{
Expliquer les évolutions des cours des matières premières agricoles : À l'impossible, nul n'est tenu !
}

\author{
Hervé GUYOMARD \\ INRA, UAR 233 Collège de direction (CODIR), \\ 147 rue de l'université, \\ 75338 Paris Cedex 07, France \\ <guyomard@rennes.inra.fr>
}

\begin{abstract}
How to explain the recent developments in the prices of agricultural raw materials? This article tries to trace the chronology of events and identify the mechanisms by which factors of change in agricultural prices act. From this analysis of temporal and causal effects an important lesson emerges, namely the difficulty if not the impossibility to isolate the responsibility of a particular determinant, even more to quantify it. Hence also the need to develop the economic analysis of agricultural prices, of their trends and determinants, including through the use of quantitative structural models, i.e., models that explicitly derived suplly and demand functions from behaviours of private and public actors.

Key words: agricultural prices, biofuels, food trade, stocks, economic analysis
\end{abstract}

aujourd'hui fleurissent. II n'y a hélas pas consensus sur les déterminants de l'évolution à la hausse, puis à la baisse, des cours agricoles ; encore moins sur les contributions respectives de chaque déterminant à cette évolution.

Les études se présentent le plus souvent sous la forme de rapports ou de notes d'institutions internationales comme la Banque mondiale (BM) ou la FAO [1], d'agences gouvernementales comme l'United States Department of Agriculture (USDA) ou la Commission européenne (CE), d'organisations professionnelles agricoles ou encore d'organisations non gouvernementales impliquées dans les questions alimentaires et/ou environnementales. Elles correspondent très rarement à des travaux scientifiques, encore moins souvent à des travaux scientifiques publiés dans des revues à comité de lecture après validation par les pairs. Ceci explique, pour partie, qu'il n'y ait pas consensus sur les explications de la flambée des cours des matières premières agricoles de la deuxième partie de l'année 2006 au début de l'année 2008, lesdites études n'étant pas toujours empreintes de la plus grande objectivité qu'au mieux nous expliquerons, sans l'excuser, par l'urgence. C'est ainsi qu'à la suite de Gohin [2], nous interpréterons la note de Mitchell [3] publiée sous la forme d'un document de travail de la BM : ce document a tendance à ne retenir que les faits et les chiffres qui permettent à Mitchell de " démontrer ce qu'il veut démontrer », à savoir la responsabilité première des politiques (ÉtatsUnis et Union européenne) de promotion des biocarburants et les restrictions aux échanges de matières premières agricoles et de biocarburants (les autres déterminants de la hausse des cours agricoles étant, selon cette étude, l'augmentation du prix de l'énergie et la déprécia- tion du dollar US). Notant qu'il y a un décalage temporel entre l'augmentation des prix agricoles et la croissance de l'activité sur les marchés financiers, Mitchell arrive à la conclusion que la spéculation n'est pour rien dans l'augmentation des prix agricoles. Conclusions toutes différentes pour Berthelot [4] qui, parmi d'autres facteurs explicatifs dont les politiques de développement des biocarburants, conclut à la responsabilité majeure de la libéralisation des échanges mondiaux agricoles et de la spéculation financière, conclusions qui lui permettent aussi « de démontrer ce qu'il veut démontrer », à savoir l'iniquité des règles du commerce mondial agricole et la responsabilité première des États-Unis et de I'Union européenne (UE) via leurs politiques agricoles (et leurs politiques de promotion des biocarburants).

Dans ce contexte, l'ambition de cet article est nécessairement modeste. II se propose de reconstruire une logique des événements en s'assurant, autant que faire se peut, de son exactitude par la reconstitution de la chronologie des faits, l'explicitation des mécanismes économiques sous-jacents et la confrontation aux chiffres. L'ambition, finalement, n'est peut-être pas si modeste!

L'article est organisé en trois sections. Après une brève présentation des principaux facteurs qui ont pu jouer à la hausse puis à la baisse sur les cours agricoles (section 1), nous nous proposons de retracer la chronologie des événements et d'identifier les mécanismes par lesquels les facteurs de variation des prix agricoles agissent (section 2). De cette analyse des enchaînements temporels et causaux, nous tirons un enseignement majeur, à savoir qu'il est difficile, si ce n'est impossible, d'isoler la responsabilité de tel ou tel déterminant, encore 
plus de la quantifier. Ce qui ne signifie pas que l'analyse économique est inutile, bien au contraire. Nous terminons alors par un plaidoyer pour une poursuite et un approfondissement de l'analyse économique des prix agricoles, de leurs évolutions et des déterminants de celles-ci, notamment en recourant à la modélisation quantitative structurelle, i.e., explicitement dérivée d'hypothèses de comportements des agents privés et publics (section 3 ).

Trois remarques avant de poursuivre. Contrairement à une idée répandue, notamment dans les médias généralistes ou spécialisés, de nombreux observateurs avaient anticipé que les prix agricoles ne pourraient durablement rester aux niveaux très bas autour desquels ils oscillaient au début des années 2000 (voir, par exemple IFPRI [5]). Comme le notent Keyzer et al. [6], "la question n'était pas de savoir si les prix allaient commencer à augmenter mais quand ». La deuxième est que ces mêmes observateurs continuent à pronostiquer que les prix agricoles seront, en moyenne, plus élevés sur au moins les dix prochaines années relativement aux niveaux du début du siècle. Reconnaissant que l'ampleur de la hausse puis de la baisse les a surpris, ces observateurs recommandent de placer l'analyse conjoncturelle des années 2006, 2007 et 2008 dans le contexte des évolutions tendancielles des offres et des demandes des différents biens agricoles. Nous adopterons cette stratégie. La troisième remarque est que certes les prix de nombreuses matières premières agricoles ont très rapidement et très fortement cru de la fin de l'année 2006 au début de l'année 2008 ; mais même quand ils étaient au plus haut, ces prix sont toujours restés inférieurs, en termes réels, aux niveaux records atteints dans les années 1970 dans le contexte des deux premiers chocs pétroliers [7]. Cette troisième remarque invite à relativiser le mouvement ici analysé de hausse puis de baisse des prix agricoles en ne le considérant pas comme exceptionnel, ce qui ne signifie pas que son impact négatif n'ait pas été dramatique pour nombre de pays en développement importateurs nets de matières premières agricoles, et dans ces pays notamment pour les ménages les plus pauvres qui proportionnellement consacrent une part plus importante de leurs (faibles) revenus à I'alimentation.

\section{Les déterminants des niveaux et des évolutions des cours agricoles : application sur la période 2006-2008}

\section{Déterminants des niveaux \\ et des évolutions des cours agricoles}

Le prix d'un bien sur un marché est le résultat d'une confrontation d'une offre et d'une demande. La simplicité apparente de cette phrase est trompeuse. Sa juste compréhension suppose en effet qu'il y ait accord sur les définitions, au minimum, du bien, du marché, de l'offre et de la demande. Dans un premier temps, supposons qu'un produit agricole donné, par exemple le blé tendre, puisse être considéré comme un bien homogène, quelle que soit sa composition, et que le marché de ce produit soit mondial, sans entraves entre échanges entre pays. Dans ce cas le plus simple, le prix du bien est mondial ; son niveau à une date donnée égalise l'offre à la demande, cette dernière étant la somme de la demande pour I'alimentation humaine, de la demande pour I'alimentation animale et des usages non alimentaires. II convient en outre de tenir compte de la variable additionnelle d'équilibre que sont les variations de stocks : le recours aux stocks des périodes précédentes revient à augmenter l'offre du moment présent et donc jouera sur le prix à la baisse, alors que le stockage d'une partie de la production de la période en cours revient à accroître la demande et par suite jouera sur le prix à la hausse ; en pratique, I'analyse économique doit alors porter sur les facteurs explicatifs du stockage/déstockage, plus précisément des comportements des organismes, publics et/ou privés, de stockage. Par ailleurs, ce cadre (très) simplifié d'analyse laisse entier le problème de la détermination des fonctions d'offre et de demande du bien considéré. L'offre du bien à une date donnée dépend de décisions adoptées antérieurement, donc d'anticipations qui ont pu être faites alors quant au prix du bien qui prévaudra ultérieurement. On peut raisonnablement supposer que les demandes réelles du bien s'ajustent plus rapidement au prix instantané (plus précisément aux variations de ce prix), et ce, de manière différenciée selon les diverses catégories de demande en fonction de leur réactivité respective au prix, i.e. en fonction des élasticités prix très vraisemblablement plus faibles (en valeur absolue) pour la demande humaine que pour la demande animale et les usages non alimentaires (sauf si ces usages non alimentaires, par exemple sous la forme de mandats $d$ 'incorporation de biocarburants, sont rendus obligatoires). En revanche, les anticipations de prix jouent très vraisemblablement un plus grand rôle dans les comportements de stockage/déstockage et de spéculation. L'anticipation que le prix du bien sera demain plus élevé peut conduire à ce que des organismes collecteurs et stockeurs retardent la mise en marché du produit, et à ce que des détenteurs de liquidités spéculent sur le marché du bien ici considéré ; le résultat sera le même, à savoir une hausse du prix instantané du bien, dans le premier cas par réduction des quantités réelles disposées sur le marché, dans le deuxième cas par augmentation des volumes demandés à la date présente même si ceux-ci, in fine, ne se traduiront pas par des échanges de quantités physiques.

Dronne, Forslund et Gohin (INRA, UMR SMART) travaillent actuellement, dans le cadre d'un contrat avec I'association PLURIAGRI, à quantifier les contributions de tel ou tel déterminant à la hausse des cours agricoles de la période allant du deuxième semestre 2006 aux premiers mois de l'année 2008, en portant un soin particulier aux effets des anticipations de prix des acteurs; leur travail repose sur l'utilisation du modèle OLEOSIM.

Poursuivons I'analyse des déterminants du prix d'un bien agricole donné en soulignant le danger, même dans le cadre des hypothèses simplificatrices adoptées ci-dessus, à ne pas tenir compte simultanément des autres produits agricoles. Dit autrement, I'analyse du mouvement considéré dans cet article de hausse puis de baisse des cours de nombreuses matières premières agricoles sur les dernières années doit être effectuée dans un cadre multiproduits de façon à rendre compte des effets de substitution entre productions/produits agricoles, aussi bien à l'offre qu'à la demande. En outre, I'analyse ne doit pas être limitée aux seuls biens agricoles : ainsi, à l'offre, convient-il de tenir compte des intrants et de leurs coûts, notamment celui de l'énergie; de même, à la demande, convient-il de replacer les évolutions des consommations des différents biens agricoles dans l'ensemble des achats des ménages, ceux-ci dépendant de multiples facteurs parmi lesquels les revenus, les prix relatifs, les changements de composition de la population et des ménages, les évolutions des habitudes de consommation par exemple sous l'effet d'une urbanisation croissante, etc.

En outre, il est clair que les hypothèses adoptées jusqu'à présent pour étudier la formation du prix d'un bien agricole donné sont trop simplificatrices. Le blé tendre cultivé dans telle zone n'est pas identique au blé tendre produit dans une autre zone. En d'autres termes, même un produit supposé simple comme le blé tendre ne peut pas être considéré comme un bien homogène et il existe autant de marchés qu'il y a de qualités de blé tendre; ces marchés de qualités différentes sont toutefois étroitement liés, en particulier parce que les substitutions à la demande entre des blés tendres de différentes qualités sont élevées. La différenciation des biens se double d'une différenciation des marchés, notamment parce que les gouvernements nationaux adoptent des politiques qui, en pratique, aboutissent à isoler, au moins pour partie, le marché domestique du marché international. Ce dernier est le plus souvent défini comme le résultat de la confrontation des excès de demande des pays importateurs et 
des excès d'offre des pays exportateurs, " une fois la demande intérieure satisfaite ». Ce marché international, que d'aucuns qualifient abusivement de résiduel, est étroit (au mieux une dizaine de points de pourcentage de la production/consommation mondiale pour les différents produits agricoles de zone tempérée) et concentré, aussi bien à l'exportation qu'à l'importation. Par suite, toute décision nationale qui aurait pour effet de diminuer les volumes d'un bien agricole mis à disposition sur ce marché mondial, par exemple sous la forme $d^{\prime}$ une taxation des exportations de façon à privilégier l'approvisionnement du marché intérieur, se traduira par une augmentation du cours mondial de ce bien, hausse qui sera d'autant plus forte, toutes choses égales par ailleurs, que le marché mondial est étroit. Un accroissement des coûts de transport, par exemple sous l'effet d'une augmentation du prix de l'énergie, aura le même impact positif sur les prix internationaux des produits agricoles par renchérissement des coûts d'approvisionnement dans les zones d'importation. II convient enfin de tenir compte des effets liés aux évolutions des parités monétaires qui, de manière générale, impactent la traduction en monnaie locale d'une évolution d'un cours mondial libellé en dollars US, aujourd'hui encore la principale monnaie de facturation des échanges, ou en euros ; cet effet comptable se double d'un effet d'entraînement, par exemple parce qu'un pays importateur net $d^{\prime} u n$ bien agricole dont la monnaie s'apprécie vis-à-vis du dollar US va augmenter ses importations, ces dernières, libellées en monnaie nationale, étant ainsi rendues moins chères ; inversement, un pays importateur net dont la monnaie se déprécie va réduire ses importations, toutes choses égales par ailleurs.

\section{Application à la période 2006-2008 : I'histoire en un coup d'œil}

Munis du cadre d'analyse présenté ci-dessus, nous sommes maintenant en position de proposer une explication d'ensemble « de ce qui s'est passé ». Nous distinguerons entre, d'une part, l'explication tendancielle de long terme sur laquelle, à de rares exceptions peu convaincantes, imprécises et partielles, pour ne pas dire partiales, il y a consensus, et, d'autre part, les facteurs explicatifs conjoncturels de court terme sur lesquels les divergences sont nettement plus nombreuses, au niveau des déterminants et encore plus de leurs responsabilités respectives. À ce stade, la présentation sera volontaire globale et succincte, l'histoire étant détaillée dans la section suivante en respectant la chronologie qui diffère selon les productions. L'objectif à ce stade est uniquement de recenser les différents facteurs explicatifs qui ont pu jouer ; il n'est pas de quantifier le poids de tel ou tel déterminant potentiel.

\section{Le contexte tendanciel}

Trostle ([8], page 6) fournit une explication globale éclairante du mouvement à la hausse des prix agricoles du début du deuxième semestre 2006 à la fin du printemps $2008^{1}$. Nous reprenons à notre compte son analyse tendancielle des facteurs structurels, à savoir depuis plusieurs années, bien avant que les prix ne commencent à augmenter, une croissance faible de la production agricole associée à une croissance forte des usages alimentaires. Cette dernière résulte de la combinaison de deux évolutions positives, soit (i) la croissance de la population mondiale et (ii) l'augmentation des consommations alimentaires individuelles générée par la croissance économique et la hausse des revenus moyens. En outre, quand les revenus individuels augmentent, les consommateurs diversifient leurs rations alimentaires en augmentant la part des huiles et la part des produits laitiers et carnés, stimulant ainsi la demande en produits animaux et par ce biais la demande en produits céréaliers et en tourteaux protéiques à des fins d'alimentation des animaux; compte tenu du fait que les ratios de transformation d'une calorie végétale en une calorie animale sont nettement supérieurs un (dans l'ordre croissant « d'inefficacité de transformation », viennent les ruminants, puis les porcins, enfin les volailles), cette modification de la composition des rations alimentaires tend à augmenter plus que proportionnellement, toutes choses égales par ailleurs, la demande en céréales et en tourteaux protéiques. Conséquence de ces évolutions contrastées, l'offre mondiale des produits de grandes cultures (céréales et oléagineux) est inférieure à la demande totale, alimentaire et non alimentaire, depuis l'année 2000 (à l'exception de l'année 2004) ${ }^{2}$. Dans ce contexte de faiblesse de l'offre relativement à la demande, les stocks mondiaux de céréales et d'oléagineux sont divisés par deux en huit années : alors qu'ils représentaient près de $30 \%$ des usages au début des années 2000, ils ne comptent plus que pour $15 \%$ de ces usages en 2008 , soit le pourcentage le plus faible depuis 1970 ([8], d'après les données de la base PSD de I'USDA). $C^{\prime}$ est dans ce contexte tendanciel non contesté qu'il convient maintenant d'examiner la

\footnotetext{
${ }^{1}$ Le rapport de Trostle [8] achevé en mars 2008, actualisé en juillet 2008, ne s'intéresse logiquement qu'à la seule phase de hausse des cours agricoles.

${ }^{2}$ Nous reviendrons plus en détails dans la deuxième section sur la question du développement des biocarburants et de son impact sur les prix des produits agricoles.
}

conjoncture qui a conduit à la hausse brutale des cours en 2006, 2007 et sur les premiers mois de 2008, puis à la baisse encore plus brutale de ces mêmes cours à compter de la fin du printemps 2008. II convient auparavant de souligner que l'évolution tendancielle via le dynamisme de la demande alimentaire, I'atonie de l'offre et la réduction progressive des stocks n'est, en quelque sorte, que la partie émergée de l'iceberg. Dans une optique prospective et normative de recommandations de politique économique, c'est aux causes de cette dualité, dynamisme de la demande alimentaire d'un côté, atonie de l'offre de l'autre, qu'il convient de s'intéresser. De même, l'origine de la réduction des stocks n'est peut-être pas uniquement imputable à un excès de demande relativement à l'offre; elle peut aussi, par exemple, résulter de décisions politiques de réduction délibérée des stocks publics.

\section{Les facteurs conjoncturels de la hausse}

Le contexte tendanciel de l'offre et de la demande décrit ci-dessus s'est accompagné d'une autre évolution à l'œuvre depuis plusieurs années et qui s'est amplifiée au début de l'année 2006. Le cours du pétrole brut, globalement stable sur la dernière décennie du $X X^{e}$ siècle, à commencé à croître au début des années 2000, de façon relativement timide dans un premier temps (de 2000 à 2003), de façon nettement plus prononcée à compter de la fin de l'année 2003 jusqu'au milieu de l'année $2008^{3}$. Cette augmentation du cours du pétrole a pour effet, toutes choses égales par ailleurs, d'accroître les coûts agricoles de production (via le renchérissement du coût de la facture énergétique directe et via l'augmentation induite du coût des intrants riches en énergie, en premier lieu les engrais) et par suite, exerce une pression à la hausse sur les prix agricoles. L'effet passe également par la hausse induite des coûts de collecte, de stockage, de transport et de distribution.

Deux autres facteurs conjoncturels situés du côté de l'offre ont également pu jouer à la hausse sur les prix agricoles internationaux, tous deux par diminution des volumes mis à disposition sur les marchés mondiaux : d'une part, les accidents climatiques des années 2006 et 2007, et, d'autre part, les décisions politiques, sur la deuxième partie de l'année 2007 et au début de l'année 2008, de plusieurs pays exportateurs nets de biens agricoles visant à res-

\footnotetext{
${ }^{3}$ Plus précisément, le cours du pétrole brut a fortement cru de la fin de l'année 2003 au milieu de l'année 2006 ; après un bref et timide repli pendant quelques mois, il a recommencé à augmenter, " de plus belle », à compter du début de l'année 2007.
} 
treindre les exportations de façon à privilégier l'approvisionnement des consommateurs domestiques ${ }^{4}$.

Du côté de la demande, quatre déterminants conjoncturels ont pu jouer, tous les quatre dans le sens d'une hausse des prix agricoles internationaux: la dépréciation, effective depuis 2002, du dollar US vis-à-vis d'un large panier de monnaies ${ }^{5}$; I'accélération de la croissance de la production mondiale de biocarburants de première génération à partir de diverses biomasses végétales (céréales, canne à sucre et betteraves à sucre, huiles végétales) ; la spéculation sur les matières premières agricoles; et les comportements « agressifs » d'achat et/ou les politiques de détaxation/défiscalisation des importations dans nombre de pays importateurs nets de produits agricoles dans I'optique d'assurer autant que possible l'approvisionnement de leurs marchés à un coût diminué.

En résumé, on retiendra que la hausse des cours mondiaux agricoles qui s'est étendue du deuxième semestre de l'année 2006 aux premiers mois de l'année 2008 résulte d'une conjonction de facteurs conjoncturels qui ont joué, (i) négativement sur les quantités mises à disposition sur les marchés internationaux (accidents climatiques et politiques restrictives à l'exportation), (ii) positivement sur les quantités demandées de par le monde (dépréciation du dollar US, développement des biocarburants de première génération, spéculation et politiques d'encouragement des importations), et (iii) positivement sur les coûts de production (augmentation du cours du pétrole, plus généralement des coûts des intrants), dans le contexte d'un mouvement tendanciel, au minimum décennal, caractérisé par l'atonie

\footnotetext{
${ }^{4}$ La situation de février 2009, date de rédaction de cet article, nous incite, par prudence, à retenir l'évolution à la hausse du cours du pétrole, plus généralement des prix des énergies fossiles, au titre des évolutions conjoncturelles. Tout en faisant nôtre la prédiction selon laquelle, sauf catastrophe économique planétaire et effondrement de la demande mondiale qu'il est impossible d'exclure, la tendance des cours des énergies fossiles sera plutôt à la hausse sur au moins les deux prochaines décennies, avec une forte variabilité temporelle.

${ }^{5}$ Les États-Unis étant un exportateur majeur de nombreux produits agricoles de zone tempérée, la dépréciation de leur monnaie a réduit le coût des importations, notamment alimentaires, des pays importateurs nets et a stimulé leur demande, notamment en biens alimentaires, adressée aux États-Unis ; elle a aussi eu pour effet d'augmenter les cours sur le marché intérieur des États-Unis et les prix, libellés en dollars US, sur le marché mondial dans un contexte où le dollar US reste la monnaie principale de facturation des échanges.
}

de l'offre, le dynamisme de la demande et la diminution des stocks.

\section{Les facteurs conjoncturels de la baisse}

La très brutale baisse des cours internationaux des produits agricoles qui est intervenue à compter du printemps de l'année 2008 s'explique alors par un retournement de conjoncture, plusieurs facteurs qui avaient pu jouer sur les prix à la hausse exerçant désormais une influence négative : climat favorable dans les diverses zones de production, appréciation du dollar US, sortie rapide des spéculateurs et baisse très brutale et très importante du cours du pétrole. Par ailleurs, les niveaux élevés des cours agricoles des années 2006 et 2007 ont incité les producteurs à augmenter les surfaces en grandes cultures au détriment des jachères ou de terres auparavant consacrées à la forêt ou à la prairie (en Amérique latine, notamment au Brésil, mais aussi en Asie du Sud-Est et en Afrique subsaharienne); ils ont également conduit les agriculteurs à chercher les rendements les plus élevés et donc à intensifier $[9,10]$. Dans ce contexte de dynamisme retrouvé de l'offre relativement à une demande, alimentaire et non alimentaire, qui est néanmoins restée soutenue, l'ajustement à la baisse des prix agricoles a permis aux pays de mettre fin à leurs politiques exceptionnelles d'encouragement des importations ou de restriction des exportations, ceci d'autant plus que ces politiques étaient budgétairement coûteuses. Retour à la normale ou accalmie de courte durée avant le retour de la tempête, i.e., le retour de la hausse des cours agricoles ? Question délicate et pourtant cruciale à propos de laquelle on se limitera ici à formuler deux observations. La première est que la conjoncture en quelque sorte favorable de l'année 2008 ne doit pas cacher que le fait que l'agriculture mondiale fait face à un triple défi d'une ampleur sans doute inégalée dans l'histoire de l'humanité : nourrir plus de 9 milliards d'individus à l'horizon 2050, l'essentiel de la croissance démographique ayant lieu d'ici 2030, dans le contexte de l'impérieuse nécessité de réduire les émissions de gaz à effet de serre (GES), de protection accrue des ressources naturelles et des biens environnementaux (sol, eau, biodiversité, etc.) et de raréfaction inéluctable des énergies fossiles [11]. La deuxième est que la baisse des cours des matières premières agricoles est intervenue avant que ne se généralise la crise financière et bancaire de cet automne, et que cette dernière se propage à la sphère réelle. Dit autrement, si la crise financière, bancaire et économique que nous connaissons depuis la fin de l'été 2008 n'est pour rien dans la baisse des prix agricoles de l'année 2008 (au bémol près que la crise du crédit hypothécaire qu'ont connu les États-Unis à compter du milieu de l'année 2007 a pu accroître le désengagement de certains acteurs financiers), ses effets sur l'agriculture (productions, demandes, mar- chés et prix) sont aujourd'hui difficiles à anticiper dans un contexte très incertain quant à l'ampleur effective de la crise, sa durée et les voies retenues pour en sortir.

\section{Un essai \\ d'explication logique : chronologie des événements et mécanismes à l'œuvre ${ }^{6}$}

Dans cette deuxième section, il s'agit, via la reconstitution de la chronologie des événements et l'explicitation des mécanismes à l'œuvre, d'analyser si les différents déterminants potentiels listés dans la première section ont effectivement joué un rôle dans le mouvement de hausse puis de baisse des prix agricoles, et d'identifier les canaux par lesquels ils ont pu jouer. II s'agit-là d'un préalable indispensable à tout essai de quantification des contributions respectives de chaque déterminant, point qui sera abordé dans la troisième section. L'attention sera plus particulièrement centrée sur les facteurs premiers à l'origine de l'évolution tendancielle de l'offre agricole et de la demande alimentaire. Elle sera aussi dirigée sur le rôle controversé de deux déterminants, d'une part, le développement des biocarburants, et, d'autre part, la spéculation sur les matières premières agricoles.

\section{L'histoire commence ainsi, au début des années 1990}

Le tableau 1 retrace l'évolution de l'indice FAO des prix alimentaires depuis l'année 2000 jusqu'au mois de janvier 2009, ainsi que celles de ses composantes regroupées en cinq agrégats : la viande, les produits laitiers, les céréales y compris le riz, les huiles végétales et animales, et le sucre ${ }^{7}$. Ce tableau montre que la hausse des cours a certes concerné les différentes catégories de produits agricoles ici distinguées, mais selon des ampleurs très variables: elle a été nettement plus importante pour les huiles,

\footnotetext{
${ }^{6}$ En pratique, l'attention sera essentiellement centrée dans cette section sur l'explication de la hausse des cours, le mouvement d'ajustement à la baisse décrit de façon synthétique dans la sous-section Application à la période 2006-2008: I'histoire en un coup d'œil n'appelant pas de compléments indispensables à la compréhension. Par ailleurs, faute de place et compte tenu de la revue, nous traiterons essentiellement des grandes cultures, sucre inclus, au détriment des produits animaux; l'analyse détaillée des évolutions de prix des produits animaux, en distinguant les produits laitiers (qui ont suivi le mouvement général de hausse et de baisse) et les produits carnés (qui sont disons restés nettement plus sages et moins volatils), nécessiterait un article spécifique.

${ }^{7}$ Voir http://www.fao.org/worldfoodsituation/ FoodPricesIndex.
} 
Tableau 1. Évolution de l'indice FAO des prix des produits alimentaires et de ses composantes.

\begin{tabular}{|c|c|c|c|c|c|c|}
\hline 100 en 2000-2004 & Indice des prix alimentaires & Viande & Produits laitiers & Céréales & Huiles et matières grasses & Sucre \\
\hline 2000 & 89 & 94 & 95 & 85 & 68 & 116 \\
\hline 2001 & 92 & 94 & 107 & 86 & 68 & 123 \\
\hline 2002 & 90 & 90 & 82 & 95 & 87 & 98 \\
\hline 2003 & 98 & 99 & 95 & 98 & 101 & 101 \\
\hline 2004 & 112 & 111 & 123 & 107 & 112 & 102 \\
\hline 2005 & 115 & 113 & 136 & 103 & 104 & 140 \\
\hline 2006 & 122 & 107 & 128 & 121 & 112 & 210 \\
\hline 2007 & 155 & 112 & 220 & 167 & 169 & 143 \\
\hline 2008 & 193 & 129 & 229 & 238 & 225 & 182 \\
\hline Janvier & 195 & 118 & 266 & 231 & 242 & 170 \\
\hline Février & 210 & 119 & 262 & 271 & 265 & 192 \\
\hline Mars & 213 & 124 & 259 & 272 & 277 & 187 \\
\hline Avril & 210 & 123 & 252 & 274 & 268 & 178 \\
\hline Mai & 211 & 132 & 250 & 267 & 271 & 171 \\
\hline Juin & 215 & 134 & 251 & 274 & 283 & 172 \\
\hline Juillet & 210 & 134 & 250 & 257 & 265 & 202 \\
\hline Août & 198 & 136 & 237 & 239 & 222 & 207 \\
\hline Sept. & 187 & 137 & 212 & 226 & 200 & 192 \\
\hline Oct. & 165 & 135 & 193 & 190 & 153 & 169 \\
\hline Nov. & 152 & 127 & 166 & 178 & 133 & 172 \\
\hline Déc. & 146 & 126 & 148 & 174 & 126 & 167 \\
\hline \multicolumn{7}{|l|}{2009} \\
\hline Janvier & 146 & 123 & 127 & 185 & 134 & 178 \\
\hline
\end{tabular}

Source : FAO (http://www.fao.org/worldfoodsituation/FoodPricelndex/fr/).

les céréales et les produits laitiers que pour la viande et le sucre. Autre point à noter, le mouvement singulier du cours du sucre : celui-ci est resté stable à un bas niveau sur la période allant de janvier 2002 au printemps 2005 ; il a fortement et rapidement augmenté pendant la deuxième partie de l'année 2005 et les premiers mois de l'année 2006 avant de redescendre, tout aussi rapidement et fortement, pendant le reste de l'année 2006 et les premiers mois de l'année 2007 ; il a de nouveau augmenté sur les trois derniers trimestres de l'année 2007 et les huit premiers mois de l'année 2008 ; il a ensuite diminué pendant les deux mois de septembre et d'octobre 2008 , et il est stable depuis cette date. De ce tableau, nous tirons deux enseignements principaux. Le premier est que la hausse des cours a affecté la très grande majorité des produits agricoles : une partie au moins de l'explication est donc commune à l'ensemble de ces biens. Le deuxième est que l'évolution spécifique du cours du sucre requiert une explication particulière $^{8}$ (tableau 1).

${ }^{8}$ Ceci est également vrai pour le cours des viandes. En outre, I'analyse au niveau des six agrégats représentés dans le tableau 1 masque des différences entre constituants de ces agrégats, par exemple entre le blé, le maïs et le riz au sein de l'agrégat céréalier.
La toile de fond : atonie de I'offre agricole et dynamisme de la demande de biens agricoles à des fins alimentaires depuis le début des années 1990

L'analyse de la première section a permis de montrer que l'envolée des cours des matières premières agricoles observée de la fin de I'année 2006 au début de l'année 2008 peut (doit) être replacée dans une perspective historique qu'il est possible de résumer par deux évolutions contrastées: d'une part, l'atonie de l'offre agricole, et, d'autre part, le dynamisme de la demande de produits agricoles à des fins d'alimentation humaine et animale. Nous ferons remonter cette double évolution tendancielle au début des années 1990. 
(i) Du côté de l'offre: du côté de l'offre, on notera que la production mondiale de céréales et d'oléagineux a cru à un rythme annuel de 2,2\% sur la période 1970-1990, mais de 1,3\% seulement sur la période 1990-2007. Ce ralentissement de la croissance de l'offre céréalière et oléagineuse est exclusivement dû au moindre dynamisme des rendements (+ 2,0\% sur $1970-1990,+1,1 \%$ sur 1990 2007) qui n'a pas été compensé par une accélération des emblavements: les surfaces en céréales et oléagineux ont cru au rythme annuel de $0,15 \%$ sur $1970-1990$, et au même taux de 0,14\% sur 1990-2007 [8]. Naturellement, ces évolutions globales masquent des disparités selon le produit et/ou la localisation : les rendements du soja ont stagné en Chine, aux États-Unis et dans I'UE-27 ; ceux du blé ont également stagné aux États-Unis et dans I'UE-27, mais ils ont augmenté en Chine ; enfin, ceux du maiis ont cru dans ces trois zones [12]. Mais constater que l'offre agricole des produits céréaliers et oléagineux a cru moins vite (relativement à la demande ou en regard du taux de croissance des décennies antérieures) ne suffit pas. II faut, dans une triple optique explicative, prospective et normative, s'intéresser aux racines de l'essoufflement de cette croissance de l'offre agricole. II faut en premier lieu s'intéresser aux causes à l'origine de la moindre croissance des rendements, voire de leur stagnation dans certaines zones depuis le début des années 2000 : affaiblissement du progrès scientifique et technique ; désintensification des processus de production sous l'influence de prix bas des produits et/ou de réglementations et contraintes environnementales plus sévères, impacts du changement climatique; etc. Autant de questions pour la recherche qui, en outre, ne saurait s'arrêter à ce stade : il faut en effet comprendre pourquoi il y aurait éventuellement eu affaiblissement du progrès scientifique et technique : par essoufflement du progrès variétal intrinsèque et/ou par insuffisance, faute de moyens, de l'effort de recherche et de recherche-développement? parce que les ressources naturelles s'épuisent et/ou parce que les réglementations environnementales sont aujourd'hui plus contraignantes ? etc. Dans le même esprit, on s'intéressera à comprendre pourquoi les terres cultivées en céréales et en oléagineux n'ont pas cru plus fortement depuis 1990 pour, en quelque sorte, compenser l'atonie des rendements : « réserves » foncières mobilisables limitées, difficiles à mettre en valeur pour des raisons topographiques (pente trop forte), climatiques (manque d'eau, températures inadaptées) ou en raison de droits de propriété défaillants ; incitations marchandes à augmenter les surfaces trop faibles; etc. (ii) Du côté de la demande alimentaire : croissance démographique, croissance économique et urbanisation expliquent le dynamisme de la consommation alimentaire depuis le début de la décennie 1990. La croissance démographique mondiale est néanmoins sur un trend décroissant depuis plusieurs décennies : $+1,7 \%$ sur les années $1975-1990,+1,5 \%$ sur la décennie 19902000 et $+1,2 \%$ sur les années 2000-2007. Ce n'est pas le cas de la croissance économique mondiale qui avait légèrement fléchi sur les années 1990-2000 mais s'est renforcée sur les années suivantes, 2000 à 2007. Les croissances sur les deux périodes sont en outre nettement plus importantes dans les pays en développement que dans les pays développés [8].Ainsi, la croissance économique chinoise se situe aux alentours des $+10 \%$ par an depuis le début des années 1990. La croissance économique indienne s'est renforcée depuis le début de ce siècle pour se situer au-delà des $+7 \%$ par an sur la période 2000-2007. Or, Chine et Inde représentent $40 \%$ de la population mondiale. Dans ces deux pays, et dans le monde en développement de façon plus générale, la croissance économique forte sur longue période a permis d'augmenter les revenus moyens par tête et, par suite, les consommations alimentaires moyennes par tête. Hausse des revenus individuels et urbanisation également à la hausse se sont en outre traduites par des modifications des compositions des paniers de biens alimentaires, au profit des produits laitiers et carnés, du poisson, des fruits et légumes, et des huiles, au détriment des céréales, des racines et des tubercules ${ }^{9}$. Ainsi, en Chine, la consommation humaine totale de céréales est $20 \%$ plus faible en 2005 qu'en 1990 ; par contraste, aux mêmes dates, celle de viandes et d'huiles est 2,4 fois supérieure, celle de lait 3 fois plus élevée et celle de fruits 3,5 fois supérieure $^{10}[13]$. La hausse des utilisations mondiales de produits laitiers et carnés a in fine un effet démultiplicateur sur la consommation totale de céréales et d'oléagineux, par l'homme et les animaux, compte tenu de coefficients de conversion des ingrédients de l'alimentation

\footnotetext{
${ }^{9}$ Le processus de modification des rations alimentaires des humains en faveur de produits à plus forte valeur ajoutée est aussi influencé par l'internationalisation croissante du secteur de la distribution de biens alimentaires.

${ }^{10}$ Keyzer et al. [6] notent ainsi que l'augmentation de la consommation chinoise de viandes, de 29 kilogrammes/an/personne en 1995 à 44 kilogrammes/ personne/an en 2005, aurait " permis ", sur la base du contenu calorique, de nourrir 300 millions de personnes en plus des 100 millions que le pays a ainsi pu alimenté en sus sur cette période de dix ans.
}

animale en un kilogramme de lait et/ou de viande nettement supérieurs à l'unité ${ }^{11}$.

Diminution des stocks mondiaux de céréales et d'oléagineux à compter du début des années 2000 ; premières augmentations des prix agricoles quelques années plus tard

Le contexte tendanciel à l'œuvre depuis le début des années 1990 est donc celui d'une demande en biens agricoles à des fins alimentaires dynamique, au minimum plus dynamique que l'offre céréalière et oléagineuse. Pourtant, quand le nouveau siècle voit le jour, les prix mondiaux des grandes cultures sont très bas $^{12}$, principalement sous l'influence d'une production abondante de façon conjoncturelle, excédentaire par rapport à la demande totale solvable : sur les cinq années 1996 à 2000, l'offre internationale de céréales et d'oléagineux est en effet supérieure aux utilisations totales, et les stocks mondiaux de ces deux agrégats de produits, en forte baisse à la fin des années 1980, puis en baisse tendancielle légère de 1990 à 1996, augmentent. En dépit de cette conjoncture de prix agricoles déprimés, les prévisions élaborées au début des années 2000 sont consensuelles (ou presque) et tablent pour une reprise à la hausse des prix agricoles: la demande alimentaire serait forte sous les effets conjugués de la démographie, de la croissance économique, de l'urbanisation et des changements dans les habitudes de consommation (cf. supra); ce "surcroît » de demande, pour une large part localisé dans les pays en développement, conduirait à un développement du commerce international agricole et à une diminution des stocks mondiaux de produits agricoles; quant aux prix mondiaux agricoles, après deux/trois années de bas étayage, ils suivraient un trend modéré à la hausse $[14,15]$.

Et c'est en pratique ce qui s'est passé ! Depuis 2000, à l'exception de l'année 2004, les utilisations annuelles totales de céréales et d'oléagineux sont inférieures à la production. Depuis 2000, les échanges de nombreux produits agricoles augmentent fortement, notamment ceux de soja, d'huile de soja, de riz, de porc, de pou-

\footnotetext{
${ }^{11}$ Ces coefficients de conversion varient selon les systèmes de production et les espèces animales (ceux des ruminants sont plus élevés que ceux des porcs, eux-mêmes plus élevés que ceux des volailles).

${ }^{12}$ En pratique, les prix mondiaux des céréales et des oléagineux ont fortement augmenté de 1992 à 1996, et encore plus fortement décru sur la deuxième partie de la décennie 1990, de sorte que, par exemple, le prix mondial du blé utilisé par le FAPRI (Food and Agricultural Policy Research Institute) pour ses analyses prospectives annuelles était d'environ 30 dollars US inférieur à la fin du siècle dernier au niveau qu'il avait sur les premières années de la décennie 1990.
} 
Tableau 2. Productions agricoles des pays exportateurs majeurs.

\begin{tabular}{|c|c|c|c|c|}
\hline $\begin{array}{l}1000 \text { tonnes } \\
\text { (\% de variation) }\end{array}$ & 2004 & 2005 & 2006 & 2007 \\
\hline Céréales ${ }^{(1)}$ & 1038325 & $1001221(-3,6 \%)$ & $932527(-6,9 \%)$ & $1041992(+11,7 \%)$ \\
\hline Oléagineux ${ }^{(2)}$ & 281589 & $293097(+4,1 \%)$ & $306387(+4,5 \%)$ & $288762(-5,8 \%)$ \\
\hline Viande ${ }^{(3)}$ & 196050 & $203317(+3,7 \%)$ & $208057(+2,3 \%)$ & $209601(+0,7 \%)$ \\
\hline Lait $^{(4)}$ & 370986 & $378730(+2,1 \%)$ & 383840 (+1,3 \%) & $394459(+2,8 \%)$ \\
\hline Sucre $e^{(5)}$ & 76882 & $93451(+21,6 \%)$ & $103101(+10,3 \%)$ & $102139(-0,9 \%)$ \\
\hline
\end{tabular}

Source : FAO (2008, page 4).

(1) Comprend l'Argentine, I'Australie, le Canada, les États-Unis, I'Inde, le Pakistan, la Thaïlande et I'UE.

(2) Comprend l'Argentine, l'Australie, le Bangladesh, le Canada, la Chine, les États-Unis, I'Inde, le Pakistan, la Fédération de Russie, I'Afrique du Sud, la Turquie et I'UE ; inclut le soja, le colza et le tournesol.

(3) Comprend l'Argentine, l'Australie, le Canada, la Chine, les États-Unis, l'Inde, la Nouvelle-Zélande, I'UE et l'Uruguay.

(4) Comprend I'Argentine, I'Australie, le Canada, les États-Unis, I'Inde, la Nouvelle-Zélande, I'Ukraine et l'UE ; production exprimée en équivalent lait.

(5) Comprend I'Australie, le Brésil, la Colombie, Cuba, le Guatemala, I'Inde, I'Afrique du Sud, la Thaïlande et I'UE.

let, etc. ; par contraste, la croissance des échanges de céréales est moindre. Depuis 2000, les stocks mondiaux de céréales et d'oléagineux diminuent; ils sont égaux à 350 millions en 2007 , soit près de 250 millions de tonnes de moins relativement au niveau de l'année $2000^{13}$. Depuis 2000 , le ratio des stocks céréaliers et oléagineux aux utilisations totales diminue de sorte qu'en 2007, il n'est égal qu'à $15 \%$, soit son plus bas niveau depuis le début des années 1970 (selon les données de I'USDA [8]). Quant aux prix, ils sont effectivement restés « déprimés » au début des années 2000 pour ensuite progressivement augmenter sur les années 2002 à 2005, avant "d'exploser» en 2006 et encore plus en 2007.

\section{L'histoire s'enrichit, à compter du début des années 2000}

Les évolutions tendancielles de l'offre et de la demande alimentaire $n$ 'auraient très vraisemblablement pas conduit à la flambée des prix agricoles du deuxième semestre 2006, de l'année 2007 et des premiers mois de 2008. D'autres facteurs ont joué, négativement sur l'offre de produits agricoles, positivement sur la demande totale en biens agricoles, ou encore positivement sur les coûts de production en agriculture (cf. section Les déterminants des niveaux et des évolutions des cours agricoles : application sur la période 2006-2008). C'est à ce titre que nous examinons ici les rôles du cours du pétrole (aug-

\footnotetext{
${ }^{13}$ Selon les données de la base PSD de I'USDA [8]. Selon les données FAO mobilisées par von Braun [13], les stocks mondiaux de céréales et d'oléagineux seraient plus élevés, les seuls stocks de céréales dépassant les 400 millions de tonnes en 2007. Néanmoins, les deux sources convergent pour aboutir à une diminution continue des stocks depuis le début des années 2000 .
}

mentation à partir du début des années 2000), du cours du dollar US (dépréciation à compter de l'année 2002), et des conditions climatiques des années 2005, 2006 et 2007.

\section{La hausse du cours du pétrole depuis 2000 et la dépréciation du dollar US depuis 2002 ont exercé une influence positive sur les prix agricoles}

Il y a consensus sur deux déterminants additionnels, à savoir (i) la hausse des prix des énergies, plus spécifiquement du cours du pétrole, et (ii) la dépréciation du dollar US, d'abord vis-à-vis de l'euro et des monnaies des pays développées, puis d'une grande majorité de monnaies y compris celles de nombreux pays en développement. Le premier déterminant a joué via un impact positif sur la facture énergétique des exploitations, le prix des engrais minéraux, mais aussi les coûts de transport, de transformation et de distribution des produits agricoles et agroalimentaires $[2,8]$. II a aussi pu jouer via le canal des biocarburants, en augmentant l'intérêt économique à utiliser la biomasse végétale à des fins de production de biocarburants en substitution du pétrole; nous détaillerons ce point ultérieurement. Dans un contexte où les ÉtatsUnis sont un exportateur majeur de nombreux produits agricoles de zone tempérée, la dépréciation du dollar US vis-à-vis d'une monnaie d'un pays importateur stimule la demande de ce pays pour les produits agricoles US et par suite, exerce une pression à la hausse sur les prix agricoles aux États-Unis et sur les prix agricoles internationaux au prorata de l'importance des exportations agricoles US dans les échanges mondiaux agricoles. En outre, comme le dollar US est aujourd'hui encore la monnaie principale de facturation des échanges agricoles, la dépréciation du dollar US étendue à un large panier de monnaies augmente les prix agricoles internationaux libellés dans cette monnaie [8]; cependant, elle affaiblit l'impact des augmentations des cours mondiaux des produits agricoles dès lors que ceux-ci sont convertis en monnaies locales [12].

S'il y a consensus sur le rôle de ces deux déterminants additionnels, impossible de quantifier leurs responsabilités respectives dans la flambée des cours sur la seule base de statistiques de quantités et de prix. Seul le recours à la modélisation contrefactuelle, via la simulation de scénarios alternatifs maintenant le prix du baril et/ou le cours du dollar aux niveaux de la fin de l'année 2005, permet d'apporter des éléments de réponse à cette question. L'exercice n'est pas dénué de risques et de pièges sur lesquels nous reviendrons dans la troisième section.

\section{De même, les conditions climatiques} défavorables des années 2005 et 2006 ont contribué à la hausse des prix agricoles

La production mondiale céréalière qui avait atteint un niveau record en 2004, a diminué de $1 \%$ en 2005 et de $2 \%$ en 2006 sous l'effet, notamment, de conditions climatiques défavorables dans plusieurs régions productrices. Elle a augmenté de plus de $5 \%$ en 2007 pour s'établir à 2,11 milliards de tonnes, sous l'influence inverse de récoltes plutôt bonnes à l'exception de la récolte australienne à un bas niveau pour la deuxième année consécutive (40 millions de tonnes en 2005, 18 millions de tonnes en 2006 et 22 millions de tonnes en 2007). La modestie des deux pourcentages de baisse des années 2005 et 2006 fait que certains analystes préfèrent concentrer leur attention sur les évolutions de la production uniquement dans certains pays exportateurs majeurs de céréales, plus généralement des différents produits agricoles de zone tempérée, sous l'argument que ce sont ces productions, et les exportations qui s'en déduisent, qui comptent puisqu'elles détermineraient les cours mondiaux dans un contexte 
tendanciel de demande plus dynamique que l'offre [16]. Ainsi, le tableau 2 reproduit les évolutions des productions de céréales (riz inclus), d'oléagineux, de viande, de lait et de sucre dans les pays exportateurs majeurs de ces produits ${ }^{14}$. Ce tableau montre que la production céréalière des huit principaux pays exportateurs ici retenus a fortement chuté en $2005(-3,6 \%)$ et en 2006 (-6,9\%), principalement sous l'effet de conditions climatiques défavorables, puis augmenté, plus fortement encore, en 2007 (+ 11,7\%). Par contraste, la production d'oléagineux des douze principaux pays exportateurs d'oléagineux ici pris en compte a d'abord augmenté (+ 4,1\% en 2005 et $+4,5 \%$ en 2006), puis diminué $(-5,8 \%)$. Or, les prix mondiaux de ces deux agrégats de produits ont suivi des évolutions similaires, c'est-à-dire une légère baisse en 2005, une première augmentation en 2006, et une deuxième augmentation nettement plus forte en 2007 (tableau 1).

Attention statistiques! Comment en déjouer les pièges, tel était le titre de l'essai de J. Klatzmann, [17] statisticien et économiste agricole, publié il y plusieurs années maintenant dans lequel il se moquait qu'un journaliste soucieux, légitimement, de la sécurité des siens avait choisi de s'installer dans l'arrondissement qui présentait le taux de délits le plus faible de la capitale et par suite, avait opté pour l'arrondissement... le moins peuplé. Plus sérieusement, le tableau 2 et le bref commentaire qui l'accompagne montrent, si besoin était, le danger des éclairages partiels : que vaut l'analyse des seuls mouvements des diverses productions agricoles, d'autant plus quand ils sont définis à partir $d^{\prime}$ une liste restreinte de pays sujette à questionnements ${ }^{15}$, si ces mouvements ne sont pas mis en regard des évolutions des demandes, des échanges et des variations de stocks (cf. première section)? Quelle que soit sa cause, une diminution des quantités disponibles d'un bien donné a pour effet mécanique, toutes choses égales par ailleurs, de rendre ce bien plus rare et donc exercera une pression à la hausse sur le prix de ce dernier. Ainsi, l'Australie a connu deux sécheresses en 2006 et 2007 qui ont entraîné une baisse de moitié de la production céréalière nationale (40 millions de tonnes en 2005, 18 millions de tonnes en 2006 et 22 millions de tonnes en 2007), baisse des volumes offerts qui a eu, sans conteste, un impact

\footnotetext{
${ }^{14}$ La liste de ces pays exportateurs majeurs varie selon l'agrégat de produits considéré ; cf. notes du bas du tableau 2.

${ }^{15}$ A titre d'illustration, le lecteur pourra s'étonner que le Brésil ne soit considéré comme un acteur majeur que pour le sucre alors que l'Argentine a droit à cette reconnaissance pour les six agrégats du tableau 2!
}

positif sur les prix mondiaux des céréales relativement à une situation contrefactuelle où la production céréalière australienne se serait maintenue au niveau de l'année $2005^{16}$. Est-il possible d'aller plus loin et de quantifier cet impact de façon incontestable? En théorie, $c^{\prime}$ est possible. II suffit en effet de déterminer de combien varie le prix mondial des céréales suite à une variation des volumes offerts; en d'autres termes et de façon résumée, il suffit de connaître l'élasticité du prix mondial des céréales relativement aux volumes offerts. En pratique, c'est nettement plus difficile parce qu'il n'y a pas consensus sur la valeur de cette élasticité ; en outre, il est plus que probable que cette valeur n'est pas constante et qu'elle varie, par exemple, en fonction des niveaux de prix, donc en fonction des quantités offertes et demandées $^{17}$; enfin, il convient de distinguer I'horizon temporel dans la mesure où l'élasticité du prix mondial par rapport aux volumes offerts est très vraisemblablement d'autant plus faible qu'elle correspond à une échéance lointaine dans le temps ${ }^{18}$.

Que retenir de l'analyse développée ci-dessus? En premier lieu, qu'il faut se méfier des statistiques partielles d'autant plus que leur emploi est parfois dicté par des objectifs partiaux. Et ce qui vaut ici pour l'offre s'applique pareillement à la demande, notamment pour ce qui est du développement des biocarburants et de la spéculation sur les matières premières agricoles (cf. infra). En deuxième lieu, l'intérêt du recours à la modélisation quantitative seule à même de pouvoir offrir un chiffrage de la contribution

\footnotetext{
${ }^{16}$ Le blé est, de loin, la principale céréale australienne. II serait donc plus exact de différencier les répercussions directes sur le marché du blé de celles qui ont lieu, par le jeu des substitutions à l'offre et à la demande, sur les marchés des autres céréales, des oléagineux, des produits animaux, etc. Mais ici la sécheresse australienne est utilisée uniquement à des fins pédagogiques de démonstration.

17 L'exemple des conditions climatiques australiennes est ici choisi à dessein dans la mesure où la baisse de la production céréalière australienne de l'année 2006 s'inscrit dans le contexte d'une récolte mondiale plutôt déprimée alors que celle de l'année 2007 fait figure d'exception dans le cadre d'une récolte mondiale plutôt bonne.

${ }^{18}$ De façon intuitive, ceci parce que son inverse, l'élasticité de l'offre par rapport au prix, croît avec le temps : faible à court terme, voire très faible dès lors que les décisions d'emblavement sont prises (l'agriculteur ne peut alors jouer que sur la seule intensification), l'élasticité de l'offre d'une culture donnée par rapport à son prix augmente dans le temps parce qu'un prix plus élevé incite les producteurs à accroître les surfaces consacrées à cette culture (on parle d'impact positif à la marge extensive) et à chercher les rendements les plus élevés (on parle d'impact positif à la marge intensive).
}

des conditions climatiques aux évolutions des cours agricoles, sous l'hypothèse additionnelle qu'il est possible de quantifier en amont l'impact de ces conditions climatiques sur les volumes offerts. L'exemple des conditions climatiques australiennes analysé ci-dessus montre, par extension, que les modèles utilisés seront des modèles multipays; au minimum, si l'intérêt est centré sur les cours dans un pays donné, il sera tenu compte de la situation mondiale et des mécanismes de transmission des variations des prix internationaux aux cours intérieurs (mécanismes influencés par les politiques commerciales et domestiques, agricoles et non agricoles). Ces modèles seront également multiproduits de façon à rendre compte des relations de substitution/complémentarité entre produits à l'offre et à la demande. Enfin, ils seront dynamiques pour rendre compte de la temporalité (cyclicité) des effets et de l'enchâ̂nement des réactions qu'ils induisent : ainsi, l'impact instantané de conditions climatiques défavorables sera de diminuer les quantités offertes et d'augmenter les cours; les prix à la hausse conduiront alors les agriculteurs à accroître les surfaces emblavées et les rendements, d'où une augmentation des volumes produits et une baisse des cours; baisse des cours qui elle-même induira une réduction des surfaces et des rendements ; etc. Dit autrement, il convient de tenir compte des effets d'entraînement dans le temps, ainsi que des effets de report sur les autres produits et les autres marchés. D'autres voies d'amélioration des modèles quantitatifs agricoles, nécessitant un effort de recherche plus que substantiel, sont présentées dans la troisième section conclusive.

\section{L'histoire se complique :}

biocarburants, spéculation

et réactions non coordonnées des états

De la même manière qu'une diminution des quantités offertes sous l'effet de conditions climatiques défavorables exerce un impact positif sur les prix, toute augmentation des volumes demandés a aussi un effet positif sur les prix (relativement à une situation contrefactuelle sans croissance des volumes demandés). Par suite, affirmons le d'emblée: le développement des biocarburants de première génération joue à la hausse sur les prix agricoles, notamment les cours des céréales du fait premier de l'expansion de la production US d'éthanol essentiellement à partir de maïs et les cours des huiles végétales par suite directe de l'expansion de la production communautaire de biodiesel essentiellement à partir de colza. La simple comparaison des volumes en cause suggère en outre que l'effet positif sur les prix lié au développement des biocarburants est au moins aussi important que celui qu'il est possible d'associer aux accidents climati- 
ques des années 2005, 2006 et 2007. L'histoire est néanmoins plus compliquée avec nécessité, ici plus qu'ailleurs, de respecter la chronologie desfaits et de différencier les produits en tenant compte des effets de substitution et de complémentarité entre ces derniers, à l'offre comme à la demande. À défaut, il sera toujours possible d'affirmer que les biocarburants ne sont pour rien dans la hausse des cours agricoles des années 2006 et 2007, et du début de l'année 2008 ; la preuve est que les cours ont fortement décru sur la deuxième partie de l'année 2008 alors que la production de biocarburants s'est maintenue ${ }^{19}$ ! Conformément à la logique qui guide nos propos depuis le début de cet article, nous dirons simplement que la conjonction d'événements qui a conduit à la hausse des cours de la fin de l'année 2006 au début de l'année 2008 diffère de celle qui a prévalu sur la deuxième partie de l'année 2008 et a engendré le reflux des cours agricoles. Dit autrement, le développement des biocarburants, notamment aux Etats-Unis et dans I'UE, a une responsabilité dans la hausse des cours parce qu'elle s'inscrit dans un contexte où d'autres facteurs ont joué simultanément dans le même sens : aux déterminants déjà mentionnés (atonie de l'offre, dynamisme de la demande alimentaire, diminution des stocks, hausse du cours du pétrole, dépréciation du dollar US et accidents climatiques), se sont ajoutées la spéculation sur les matières premières agricoles (en 2007 et sur les premiers mois de l'année 2008) et les réactions désordonnées des états (sur la deuxième partie de l'année 2007 et les premiers mois de

\footnotetext{
${ }^{19}$ En support à un tel jugement, quelques citations : "La baisse [sur la deuxième partie de l'année 2008] du prix des céréales, alors que la production d'éthanol est en hausse, prouve bien que les agrocarburants n'étaient pour pas grand-chose dans l'envolée des cours l'an passé. " [9]; "Les prix du riz ont été multipliés par trois alors que pas un litre d'éthanol n'est tiré de cette céréale. " [18]; "En définitive, les argumentaires qui ont fait porter la responsabilité de la hausse des prix aux biocarburants semblent d'autant plus excessifs maintenant que le reflux des prix a commencé et que la production de biocarburants continue. » [19]. Courleux [19] mobilise notamment une étude de l'OCDE [20] qui montre, sur la base d'une analyse statistique des relations de causalité entre prix, que le prix du pétrole aurait exercé une influence significative sur le cours du sucre, de l'éthanol et de l'huile de soja, mais n'aurait eu aucun impact significatif sur le prix du maïs. Toujours selon cette étude, c'est le prix du blé qui aurait influencé celui du maïs, et non I'inverse. Courleux utilise ces résultats comme argument additionnel "pour disculper les biocarburants dans la hausse du prix des céréales ». Précisons immédiatement que la position contraire accusant les biocarburants de tous les maux (i.e., d'être la principale cause d'augmentation des cours agricoles) est naturellement tout aussi excessive.
}

I'année 2008). À compter du deuxième trimestre 2008, ces mêmes déterminants ont joué sur les cours agricoles à la baisse, en quelque sorte par inversion des évolutions (offre agricole à la hausse en réponse aux prix agricoles élevés et dans le contexte de conditions climatiques plutôt favorables, légère croissance des stocks, baisse du cours du pétrole, légère appréciation du dollar US). Tous les déterminants se sont ainsi inversés sauf deux, la demande alimentaire et la production de biocarburants qui sont restées dynamiques sur l'année 2008 : nul doute que le maintien d'une forte demande alimentaire et non alimentaire ait contribué à ce que la baisse des cours de l'année 2008 ne soit pas plus accentuée encore.

\section{On fera remonter à l'année 2003 l'entrée en scène des biocarburants, à l'été 2005 ses premiers effets prix (impact positif du développement du bioéthanol US sur le cours mondial du sucre)}

En 2003, la production mondiale de bioéthanol se partageait à parts égales entre le Brésil et les États-Unis. Alors que le Brésil a recours à la canne à sucre comme matière première végétale, les États-Unis utilisent le maïs. C'est à compter de cette date que la production US de bioéthanol a commencé à croître significativement sous I'influence de facteurs économiques, environnementaux et politiques (hausse du cours du pétrole; volonté de réduire la dépendance énergétique à l'égard du pétrole ; volonté de développer des énergies présentant un bilan environnemental, principalement en matière d'émissions de GES, plus favorable que le pétrole ; obligations et incitations fiscales à l'utilisation de bioéthanol ; etc.). Les ÉtatsUnis sont aujourd'hui le premier producteur mondial de bioéthanol $(6,49$ milliards de gallons en 2007), juste devant le Brésil (5,28 milliards de gallons), loin devant les troisième et quatrième producteurs (I'UE-27 avec 488 millions de gallons et la Chine avec 469 millions de

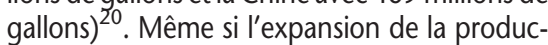
tion mondiale de bioéthanol depuis 2003 n'est pas limitée aux seuls États-Unis [8], le rôle premier de ce pays dans ce développement invite clairement à « tirer le fil de la pelote » en commençant par analyser comment les États-Unis ont pu augmenter leur production de bioéthanol, et l'impact de cette production augmentée sur les utilisations des diverses matières premières agricoles, le maïs en premier lieu.

${ }^{20}$ La production communautaire de bioéthanol a recours au blé (à hauteur de $46 \%$ en 2007 ; [8]), à la betterave à sucre (à hauteur de $29 \%$ ) et aux importations de diverses matières végétales pour transformation domestique en bioéthanol (à hauteur de $25 \%$ ). Par ailleurs, I'UE (plus spécifiquement, certains États membres comme la Suède) est un importateur net de bioéthanol.
C'est aussi à compter de 2003 que la production mondiale de biodiesel a commencé à augmenter de façon substantielle. Jusqu'à cette date, il n'y avait qu'un seul producteur significatif au monde, I'UE qui avait principalement recours à l'huile de colza comme matière première végétale. Depuis lors, la production communautaire de biodiesel a fortement augmenté (multiplication par trois entre 2004 et 2008) sous l'influence première d'une volonté politique forte, notamment via la fixation d'objectifs d'incorporation ambitieux (actuellement, I'objectif communautaire est une incorporation de $10 \%$ de biocarburants dans les carburants utilisés pour le transport routier à l'horizon 2020). La production communautaire de colza (ainsi que de tournesol) ne suffisant pas, l'UE doit importer, et devra importer d'autant plus qu'elle achèvera un taux élevé d'incorporation, des graines oléagineuses, des huiles végétales et du biodiesel, ce dont profitent (devraient profiter) de nombreux pays tels que la Russie, I'Ukraine, I'Argentine, I'Indonésie, etc. (du moins tant que les seuls biocarburants de première génération seront mobilisés). C'est donc en partant de la situation dans I'UE et de la manière dont cette entité géographique est parvenue à augmenter sa production de biodiesel que nous allons tenter de dévider la pelote. Ce serait néanmoins une double erreur de raisonner séparément en considérant, d'une part, le couple bioéthanol et mais aux États-Unis, et, d'autre part, le couple biodiesel et colza dans I'UE. En premier lieu parce que les États-Unis ont également développé une production domestique de biodiesel, et I'UE une production locale de bioéthanol (cf. note de bas de page $n^{\circ} 20$ ). En deuxième lieu, et de façon plus importante, parce que l'expansion de la sole US de maïs pour transformation en bioéthanol, notamment en 2007, s'est pour une large opérée au détriment de la surface et de la production de soja dans ce pays, avec donc des répercussions directes sur cet oléagineux et, par le jeu des substitutions et des complémentarités, sur l'ensemble des huiles végétales. Nous voici prêts à retracer la logique et la chronologie d'action des biocarburants sur les prix agricoles. Plaçons-nous au début de l'année 2003 dans le contexte déjà décrit d'atonie de l'offre de grandes cultures, de dynamisme de la demande alimentaire et des premières diminutions des stocks de céréales et d'oléagineux. Le cours du pétrole est à la hausse depuis quelques années, et le dollar commence à se déprécier vis-à-vis d'un panier croissant de monnaies. La production mondiale de biocarburants est à la hausse, essentiellement aux États-Unis sous la forme de bioéthanol. Le recours aux stocks et la récolte céréalière et oléagineuse record de l'année 2004 permettent de contenir les augmentations des cours céréaliers et oléagineux pendant les trois années 2003, 2004 et 2005; mieux, les prix internationaux de ces deux grou- 
pes de produits diminuent légèrement en 2005. Au début de l'année 2004, l'augmentation du cours du pétrole s'accélère et le dollar continue à se déprécier. Les stocks de clôture céréaliers et oléagineux qui avaient pu légèrement croître en 2004 sont à nouveau à la baisse en 2005 et 2006, deux années pendant lesquelles les conditions climatiques sont en outre plutôt défavorables et la production mondiale céréalière à la baisse $(-1 \%$ en $2005,-2 \%$ en 2006). Dans un contexte où les stocks US de maïs sont à la baisse et la production US de maïs insuffisamment dynamique, faute de prix suffisamment incitatifs, les États-Unis, dans l'incapacité de satisfaire leur législation en matière d'incorporation de bioéthanol, se tournent vers l'étranger, plus spécifiquement vers le Brésil : le prix international du sucre explose en huit mois, passant de l'indice 128,4 en juin 2005 à l'indice 189,3 en décembre 2005 (+ $47 \%$ en six mois), puis à l'indice 254,3 en février 2006 (+ $34 \%$ en deux mois). En réponse à ces prix élevés du sucre (et de l'éthanol), les surfaces brésiliennes consacrées à la canne à sucre augmentent. Le maïs US va progressivement prendre le relais du sucre brésilien. Par suite, le prix international du sucre va amorcer un reflux à compter du mois de mars 2006, passant à l'indice 218,5 en juin 2006 et à l'indice 164,2 en décembre 2006 ; la baisse va se poursuivre sur toute l'année 2007 et ce n'est qu'au début de l'année 2008 que les cours mondiaux du sucre repartiront à la hausse, en quelque sorte dans le cadre du mouvement global $d^{\prime}$ augmentation des prix agricoles.

Dans un contexte de stocks mondiaux au plus bas, le développement des biocarburants aux États-Unis et dans I'UE va jouer à la hausse sur les cours des céréales et des huiles végétales à compter du deuxième trimestre de l'année 2006, dans un contexte de stocks mondiaux au plus bas ${ }^{21}$

Voilà décrite I'histoire spécifique du prix du sucre dont l'évolution sur les années 2005, 2006 et 2007 est donc étroitement liée au développement des biocarburants, plus spécifiquement au développement du bioéthanol aux États-Unis. Ce déterminant a joué sur le prix du sucre, à la hausse dans un premier temps (sur l'année 2005 et les premiers mois de l'année 2006 via un effet de demande accrue), à la baisse dans un deuxième temps (sur le reste de l'année 2006 et toute l'année 2008 dans un contexte d'offre de sucre excédentaire, le maïs US remplaçant progressivement le sucre brésilien). Ce même déterminant va jouer de façon identique sur le cours international du maïs, contribuant, dans un premier temps à la hausse du cours mondial de cette céréale à compter des derniers mois de l'année 2006 jusqu'au pre-

\footnotetext{
${ }^{21}$ Stocks au plus bas en partie liée au développement des biocarburants!
}

Tableau 3. Utilisations du maïs US pour la fabrication de bioéthanol, 2001/02 à 2007/08.

\begin{tabular}{|lll|}
\hline & \multicolumn{2}{l}{ Utilisations du maïs US pour la fabrication de bioéthanol } \\
\cline { 2 - 3 } & En millions de tonnes & En \% de la production \\
\hline $2001 / 02$ & 17,9 & $7,4 \%$ \\
\hline $2002 / 03$ & 25,3 & $11,1 \%$ \\
\hline $2003 / 04$ & 29,7 & $11,6 \%$ \\
\hline $2004 / 05$ & 33,6 & $11,2 \%$ \\
\hline $2005 / 06$ & 40,7 & $14,4 \%$ \\
\hline $2006 / 07$ & 53,8 & $20,1 \%$ \\
\hline $2007 / 08$ & 76,2 & $22,9 \%$ \\
\hline
\end{tabular}

Source : http://www.ers.usda.gov/Data/Feedgrains/Standard/Reports/YBtable4.htm.

mier trimestre de l'année 2008, dans un deuxième temps à la baisse de ce prix à partir du deuxième trimestre de l'année $2008^{22}$.

En pratique, les États-Unis cherchent à favoriser la production nationale de bioéthanol à partir de ressources végétales domestiques, en d'autres termes à partir de maïs. Le tableau 3 illustre ainsi la croissance des utilisations de maïs US pour la production de bioéthanol aux États-Unis sur la période 2001/02 à 2007/08 : en 2003/04, la quantité de maïs US utilisée pour la fabrication de bioéthanol représentait un peu moins de 30 millions de tonnes, soit 11,6\% des utilisations totales du maïs US; en 2006/07, le même usage représentait 53,8 millions de tonnes $(20,1 \%)$ et en $2007 / 08$, il s'établissait à 76,2 millions de tonnes (22,9\%). Ces quelques statistiques suggèrent une responsabilité première du développement du bioéthanol aux États-Unis sur le mouvement à la hausse du prix du maïs dans ce pays. Dans la mesure où les États-Unis sont, et de très loin, le premier exportateur mondial de maïs, le mouvement de hausse du prix US de cette céréale s'est rapidement étendu à son prix international.

Le développement du bioéthanol aux ÉtatsUnis a donc eu pour effet premier d'augmenter le cours mondial du sucre, du début de l'été 2005 aux premiers mois de l'année 2006 (cf. supra). Le remplacement progressif du sucre brésilien par le maïs US s'est d'abord opéré en mobilisant les stocks de maïs US, puis en augmentant les surfaces US emblavées en maïs. Le développement du bioéthanol aux ÉtatsUnis a donc eu pour deuxième effet $d$ 'augmenter le cours international du maïs (et incidemment, de provoquer simultanément l'ajustement à la baisse du cours mondial du sucre, en quelque sorte par excès d'offre de sucre).

\footnotetext{
${ }^{22}$ En passant, le lecteur notera avec intérêt que le Brésil est le premier exportateur mondial de sucre et d'éthanol, et que les États-Unis sont le premier exportateur mondial de maïs.
}

C'est lors de la campagne 2006/07 que l'augmentation du cours mondial du maïs fut la plus importante (50\% environ) ; par comparaison, les hausses des prix internationaux du blé et du riz furent deux fois moindres lors de cette année campagne. Le différentiel de prix favorable au maïs relativement aux autres céréales et aux oléagineux a incité les producteurs, en premier lieu les producteurs US, à augmenter les surfaces consacrées au maïs, notamment lors de la campagne 2007/08: les surfaces mondiales cultivées en maïs qui avaient diminué de $3 \%$ sur la période 1996/97 à 2002/3, ont augmenté de $14 \%$ sur la période 2002/03 à 2007/08, et l'essentiel de cette hausse (environ la moitié, soit $7 \%$ ) a eu lieu lors de la campagne 2007/08, pour une large part au détriment de la sole oléagineuse qui en une seule campagne a décru de $4 \%[21]^{23}$. Nul doute que cette diminution de la sole mondiale oléagineuse en 2007/08, en premier lieu aux ÉtatsUnis, ait contribué à exercer une pression additionnelle à la hausse sur le cours des graines oléagineuses et des diverses huiles végétales sur cette campagne 2007/08: entre juin

\footnotetext{
${ }^{23}$ Mais la surface mondiale cultivée en graines de soja avait cru de près de $15 \%$ entre 2002/03 et 2006/07, essentiellement en Amérique du Sud pour satisfaire la demande chinoise en forte croissance : depuis le milieu des années 1980, la croissance de la consommation chinoise d'huiles végétales a en effet été supérieure à la croissance de la production domestique, différentiel qui fait que la Chine importe aujourd'hui (2007) environ 10 millions de tonnes d'huiles végétales, dont 6 millions de tonnes d'huile de palme et 3 millions de tonnes d'huile de soja [21]. Comment interpréter cette observation ? À nouveau en soulignant la nécessité de ne pas se focaliser à l'excès sur l'analyse d'un déterminant considéré isolément en veillant à positionner ce déterminant et son influence dans le contexte d'ensemble qui définit les conditions d'évolution des offres et des demandes des différents biens agricoles.
} 
2007 et juin 2008, I'indice FAO du prix mondial des huiles a ainsi augmenté de près de $79 \%$; par comparaison, sur la même période, les prix mondiaux du maïs ont cru d'un peu plus de $20 \%$ seulement, ceux du riz d'un peu moins de $50 \%$ et ceux du blé d'un peu plus de $90 \%$. Le développement du bioéthanol aux États-Unis a donc eu pour troisième effet, lors de la campagne 2007/08, d'augmenter la sole mondiale (US) de maïs au détriment de la sole mondiale (US) de soja, ceci en réaction au différentiel de prix de l'année passée favorable au maïs relativement aux graines oléagineuses (soja), et aussi par anticipation que la demande de maïs resterait ferme, développement du bioéthanol US oblige. Cet effet de substitution à l'offre favorable au maïs et défavorable au soja a contribué à accroître la pression à la hausse sur le prix des graines de soja et de l'huile de soja, et par extension sur les diverses huiles végétales fortement substituables à la demande ${ }^{24}$. Cet effet de contagion sur les prix des diverses huiles végétales a été $d^{\prime}$ autant plus fort qu'il s'est inscrit dans le contexte plusieurs fois rappelé mais qu'il ne faut pas oublier de plusieurs facteurs jouant tous dans le sens d'une augmentation générale des cours agricoles (atonie de l'offre, dynamisme de la demande alimentaire, diminution des stocks, hausse du cours du pétrole et dépréciation du dollar US). Dans le cas spécifique des huiles végétales, ajoutons le développement concomitant de la production communautaire de biodiesel qui a été un facteur additionnel de pression à la hausse, significatif compte tenu des tonnages représentés, sur les cours des huiles végétales, en premier lieu le prix du colza.

Plusieurs études se sont attachées à quantifier la responsabilité des biocarburants dans la hausse des cours agricoles de la deuxième partie de l'année 2006, de l'année 2007 et de la première partie de l'année 2008. Les différences en termes de données, de méthodologies, de scénarios, d'horizons des simulations, etc. rendent la comparaison des résultats délicate. Néanmoins, à de (très) rares exceptions, un enseignement émerge : une responsabilité forte du développement du bioéthanol aux États-Unis dans le mouvement de hausse, en premier lieu du prix du maïs et par effet de report, via le jeu des substitutions à l'offre et à la demande, des prix des diverses céréales et des différentes huiles (y compris, par exemple dans l'étude de I'IFPRI réalisée à I'aide du modèle IMPACT, du prix du

\footnotetext{
${ }^{24}$ Selon l'OCDE [20], le cours du pétrole exercerait une influence statistique significative sur les cours des huiles de soja et de palme (au seuil de significativité de $5 \%$ ), ainsi que sur l'huile de colza (à un seuil de significativité moindre, uniquement à partir de $10 \%)$. Et le cours de l'huile de soja exercerait une influence, au seuil de significativité de $5 \%$, sur les prix des huiles de colza et de palme.
}

riz bien que cette céréale ne soit pas utilisée pour produire du bioéthanol). Sur la base d'un recensement de plusieurs études et de travaux additionnels ad hoc, Collins [22] arrive ainsi à la conclusion qu'entre 25 à $50 \%$ de l'augmentation du cours US du maïs entre les campagnes 2006/07 et 2008/09 pourrait être attribuée à la croissance du débouché de l'éthanol dans ce pays. On regrettera ici le nombre insuffisant d'analyses, du moins à notre connaissance, centrées sur la seule mesure des effets prix du développement des biocarburants dans la seule UE, les études étant focalisées sur le développement des biocarburants dans les seuls États-Unis ou dans l'ensemble du monde (voir, néanmoins, [7] ou l'étude en cours déjà mentionnée développée par Dronne, Forslund et Gohin).

La responsabilité de la spéculation financière dans le mouvement de hausse 2007/08 des cours des matières premières agricoles serait faible, voire nulle; les analyses sur ce point nécessitent d'être poursuivies et approfondies

À de rares exceptions (par exemple, Berthelot [4]) la littérature existante conclut que la spéculation financière sur les matières premières agricoles n'aurait pas eu d'impact significatif sur le mouvement de hausse des cours agricoles, notamment dans la dernière phase 2007/08 d'augmentation.

Cette spéculation financière aurait eu pour effet premier d'accroître la volatilité des cours ${ }^{25}$, mais elle ne serait pas à l'origine de la hausse de ces prix dans la mesure où il n'a pas été possible de démontrer, de façon statistiquement robuste, que les marchés financiers influenceraient les fondamentaux de l'offre et de la demande [22-24].

Un peu plus prudents, Keyzer et al. [6] notent simplement que "sur la base de la recherche existante, il est impossible de dire si les niveaux de prix [quand ils étaient à la hausse] ont été influencés par l'activité spéculative". Même conclusion pour Courleux [19] : "Si la spéculation ne peut pas créer une tendance, elle est susceptible de l'amplifier. Pour autant, les informations disponibles sur les marchés réglementés ne permettent pas de quantifier l'effet de la spéculation dans le mouvement de hausse. En particulier, le procès intenté aux fonds indiciels semble exagéré. Les liens entre la crise financière et la flambée des matières premières sont difficiles à mettre en évidence ». Un pas de plus pour Banse et al. [7] qui constatent qu'à la rédaction (juin 2008) de leur rapport, les prix sur les marchés à terme sont plus élevés que les prix spot, ceci à cause d'une anticipation partagée à la hausse; par

${ }^{25}$ À titre d'illustration, Banse et al. [7] notent ainsi
que la volatilité du cours du maiis pendant la pre-
mière semaine du mois de mars 2008 a dépassé les
150 dollars US par tonne, une fluctuation supérieure
au prix moyen du maïs observé l'année précédente. suite, une partie de la hausse actuelle (i.e., celle du premier semestre de l'année 2008) pourrait être attribuée à cette « bulle spéculative », partie que Banse et al. se refusent néanmoins à quantifier.

Le lecteur pourra s'étonner de la brièveté de notre analyse quant au rôle potentiel de la spéculation dans le mouvement de hausse des cours agricoles. Mais prudence est mère de sureté. En conséquence, nous avons ici renoncé à entrer dans une description détaillée du fonctionnement des marchés financiers, plus spécifiquement en ce qu'ils concernent et impactent les matières premières agricoles. Plusieurs auteurs ont souligné la difficulté d'une telle analyse, notamment pour des raisons d'inadaptation des données statistiques en relation avec les différents acteurs, et leurs motivations, qui interviennent sur les marchés financiers [7, 10]. Et reconnaissons le humblement : I'auteur de ces lignes, au mieux « spécialiste » de l'analyse des marchés réels des matières premières agricoles, ne possède pas toutes les connaissances et compétences pour s'autoriser un avis définitif quant à la responsabilité possible de la spéculation financière sur le mouvement de hausse des cours agricoles. Dans la mesure où cette remarque s'applique aussi à de nombreux économistes agricoles disons « traditionnels ", nous terminerons ici par un appel à la poursuite de l'analyse via la confrontation de deux communautés d'économistes, d'une part, les modélisateurs des marchés agricoles, et, d'autre part, les économistes spécialistes des marchés financiers (tout en ne sous-estimant pas la difficulté de la tâche compte tenu, notamment, des différences en termes d'objectifs, d'outils d'analyse, etc.).

Les réactions unilatérales des États visant à privilégier le marché domestique ont amplifié la hausse des cours agricoles des céréales et des huiles végétales lors de la campagne 2007/08 ; elles ont joué un rôle majeur

dans l'envolée du cours du riz sur cette période L'augmentation des cours mondiaux de nombreuses matières premières agricoles, en premier lieu ceux des céréales, riz inclus, de la fin de l'année 2006 et du début de l'année 2007 ont vite conduit à des augmentations des prix alimentaires dans la totalité des pays, dans des proportions néanmoins généralement plus faibles compte tenu de l'imparfaite transmission des prix internationaux aux prix intérieurs et de l'isolement partiel de nombreux marchés domestiques [6]. Ces augmentations des prix alimentaires ont toutefois été vite insupportables dans de nombreux pays en développement, d'autant plus qu'ils dépendaient du marché mondial pour leur approvisionnement. De façon générale, de très nombreux pays ont adopté, à compter du troisième trimestre de l'année 2007 jusqu'au milieu de l'année 2008, des mesures visant, dans le cas des pays exportateurs à décourager les exportations de 
Tableau 4. Mesures non coordonnées des pays, à l'exportation et à l'importation, en réaction à la hausse des prix agricoles (campagne 2007/08).

Mesures à l'exportation (à compter de l'automne 2007)

Élimination des subventions à l'exportation (céréales et produits des céréales en Chine)

Taxes à l'exportation (céréales et produits des céréales en Chine ; céréales et produits oléagineux en Argentine ; blé en Russie et au Kazakhstan ; huile de palme en Malaisie et en Indonésie)

Restrictions quantitatives à l'exportation (blé en Argentine ; blé en Ukraine ; riz en Inde et au Vietnam)

Interdictions d'exportations (blé en Ukraine, Serbie et Inde ; riz en Egypte, au Vietnam, au Cambodge et en Indonésie ; certaines variétés de riz en Inde, troisième exportateur mondial)

Mesures à l'importation (à compter du début de l'année 2008)

Réduction des droits de douane (farine de blé en Inde ; blé et soja en Indonésie ; blé en Serbie ; porc en Thaillande ; céréales dans I'UE, différents produits agricoles en Corée du Sud et en Mongolie)

Subventions à la consommation (Maroc, Venezuela, etc.)

Source : Trostle (2008).

façon à privilégier l'approvisionnement du marché domestique, dans le cas des pays importateurs à diminuer les coûts d'achat sur les marchés internationaux. Le tableau 4, tiré de [8], liste ces réactions non coordonnées des états qui, au total, ont contribué, en raréfiant l'offre d'exportation et/ou en augmentation la demande d'importation, à amplifier la hausse des cours agricoles, en premier lieu ceux des céréales et des huiles végétales.

À notre connaissance, une seule étude, celle du ministère canadien en charge de l'agriculture (non publiée, présentée dans [7] sous la forme d'un tableau de synthèse), a essayé de quantifier les impacts de ces politiques unilatérales des états sur les prix mondiaux. II apparaît que les effets de ces mesures sur la hausse des cours de la campagne 2007/08 seraient faibles (aux alentours de $2 \%$ pour le blé et le maïs, moins de $1 \%$ pour les graines oléagineuses et les huiles végétales), à l'exception du riz : les politiques non coordonnées de restriction des exportations et d'encouragement des importations auraient conduit à augmenter le cours mondial du riz de la campagne 2007/08 de $7 \%$ additionnels. Faute d'informations détaillées, il est très difficile, pour ne pas dire impossible, d'apprécier la robustesse de ces conclusions. On se permettra seulement d'associer, sous forme d'hypothèse, la plus forte réactivité du cours du riz à l'étroitesse du marché mondial de ce produit ${ }^{26}$. En support à cette hypothèse, Headey et Fan [24] notent la corrélation temporelle entre les décisions de restriction des exportations/d'encouragement des importations et la hausse du prix international du riz à

\footnotetext{
${ }^{26}$ Sur les cinq dernières années, les pourcentages de production échangés sur les marchés mondiaux sont d'environ $7 \%$ pour le riz, $12 \%$ pour le maïs et $18 \%$ pour le blé [24].
}

compter du mois de novembre 2007 jusqu'au mois de mai 2008, date à laquelle le Japon accepta de livrer aux Philippines 200000 tonnes de riz qu'il détenait dans ses stocks: du mois d'août 2005 au mois de novembre 2007, le prix mondial du riz a augmenté de $50 \%$; du mois de novembre 2007 au mois de mai 2008, il a été multiplié par plus 2,5 ; et il a baissé dès le mois de juin 2008 suite à la décision du Japon, imité quelques semaines plus tard par le Cambodge. En résumé, une hypothèse plausible, qui mérite néanmoins d'être confortée par des analyses statistiques complémentaires, est que les réactions non coordonnées des états sur la deuxième partie de l'année 2007 et les premiers mois de l'année 2008 sont une cause première de l'envolée des cours internationaux du riz sur cette période. Ceci parce que le marché mondial du riz est étroit et les politiques de restriction des exportations et d'encouragement des importations ont été mises en œuvre par des acteurs majeurs sur le marché du riz, I'Inde en premier lieu. Pour les mêmes raisons, mais cette fois jouant en creux (marchés mondiaux plus développés, politiques mises en œuvre par des acteurs de moindre importance en termes de volumes produits, consommés et échangés), les politiques unilatérales des états auraient eu un impact moindre sur les prix internationaux des autres céréales et des huiles végétales sur la campagne 2007/08 (tableau 4).

\section{L'impérieuse nécessité de poursuivre les analyses économiques quantitatives}

Àl'issue de l'analyse logique (chronologique et causale) des événements qui ont conduit à la flambée des cours de nombreuses matières premières agricoles à compter de la deuxième moitié de l'année 2006 jusqu'au printemps de l'année 2008, puis à la baisse très brutale de ces mêmes cours sur le deuxième trimestre de I'année 2008, deux enseignements émergent clairement, du moins selon nous. Ces enseignements feront office de conclusions.

Le premier enseignement est que la hausse des cours est due à un faisceau de facteurs qui ont joué simultanément dans le même sens. Le tableau 5 de synthèse reprend ces différents facteurs en les classant en trois catégories: (i) les déterminants tendanciels à l'œuvre depuis le début des années 1990, (ii) les déterminants additionnels à l'œuvre depuis le début des années 2000, et enfin (iii) les déterminants conjoncturels qui ont joué sur la période où la hausse latente des cours des céréales et des huiles végétales s'est transformée en flambée. Les évolutions de plusieurs de ces facteurs se sont inversées à la fin du printemps de l'année 2008 , et ont conduit à la correction forte et rapide des prix à compter de cette date : en pratique, la demande alimentaire et non alimentaire est certes restée dynamique, mais tous les autres facteurs ont joué dans le sens d'un ajustement à la baisse des cours agricoles de céréales et d'oléagineux ${ }^{27}$ : I'offre s'est raffermie, à la marge extensive (augmentation des surfaces) et à la marge intensive (augmentation des rendements), pour une large part en réponse aux incitations fournies par les prix agricoles élevés des deux années antérieures ; les stocks se sont (un peu) reconstitués; le dollar US a cessé de se déprécier, voire s'est apprécié vis-à-vis de nombreuses monnaies; le cours de pétrole a très rapidement et très fortement diminué, dans une ampleur que peu d'analystes avaient anticipé; l'année 2007/08 fut plutôt une bonne année climatique ; la crise financière, bancaire et aujourd'hui économique a conduit à un désengagement des investisseurs financiers des marchés des matières premières agricoles dans un contexte de besoin aigu de liquidités; et enfin, la baisse des prix agricoles a permis aux états d'assouplir, souvent d'abandonner, leurs politiques de restriction des exportations et d'encouragement des importations (également parce que ces politiques étaient budgétairement coûteuses) (tableau 5).

Dans cet ensemble, le sucre et le riz peuvent apparaître comme deux produits singuliers. Le sucre parce que la première hausse de son cours international a eu lieu très tôt, une année avant la hausse quasi-générale; nous avons proposé une explication propre de cette singularité en l'associant au développement du bioéthanol aux États-Unis qui n'a pu, dans un premier temps, être satisfaite par le maïs

\footnotetext{
${ }^{27}$ Ainsi qu'à la baisse des prix internationaux des produits laitiers.
} 
Tableau 5. Les facteurs d'évolution à la hausse des cours agricoles à l'œuvre sur la période allant du deuxième semestre de l'année 2006 à la fin du printemps de l'année 2008.

\begin{tabular}{|c|c|}
\hline Facteurs & Mécanismes \\
\hline \multicolumn{2}{|l|}{ Tendances depuis 1990} \\
\hline - Atonie de l'offre (depuis 1990) & - Impact négatif sur l’offre \\
\hline $\begin{array}{l}\text { - Dynamisme de la demande alimentaire } \\
\text { (depuis 1990) }\end{array}$ & - Impact positif sur la demande \\
\hline \multicolumn{2}{|l|}{ Évolutions à I'œuvre depuis les années 2000} \\
\hline $\begin{array}{l}\text { - Développement des biocarburants, notamment } \\
\text { aux États-Unis et dans I'UE, sous le double effet de } \\
\text { politiques ambitieuses en ce domaine et d'incitations } \\
\text { privées via la hausse du cours du pétrole } \\
\text { (depuis 2003) }\end{array}$ & - Impact positif sur la demande \\
\hline $\begin{array}{l}\text { - Diminution des stocks, pour partie fruit des } \\
\text { évolutions tendancielles de l'offre et de la demande, } \\
\text { pour partie résultat de décisions politiques visant à } \\
\text { diminuer les stocks publics (depuis 2000) }\end{array}$ & - Impact négatif sur les disponibilités \\
\hline - Hausse du cours du pétrole (depuis 2000) & $\begin{array}{l}\text { - Impact positif sur les coûts de production, donc sur } \\
\text { les prix; impact aussi sur les coûts de stockage, } \\
\text { transport et distribution }\end{array}$ \\
\hline - Dépréciation du dollar US (depuis 2002) & - Impact positif sur les cours mondiaux en dollar US \\
\hline \multicolumn{2}{|l|}{$\begin{array}{l}\text { Déterminants additionnels des années } 2006 \\
\text { et 2007, et du début de I'année } 2008\end{array}$} \\
\hline - Accidents climatiques (2006 et 2007) & - Impact négatif sur l'offre \\
\hline $\begin{array}{l}\text { - Spéculation sur les matières premières agricoles } \\
\text { (à compter du l'été 2007) }\end{array}$ & - Impact positif sur la demande \\
\hline $\begin{array}{l}\text { - Réactions unilatérales des états, via des restrictions } \\
\text { à l'exportation et des encouragements } \\
\text { à l'importation (à compter de l'automne 2007) }\end{array}$ & $\begin{array}{l}\text { - Impact négatif sur l'offre d'exportation et positif sur } \\
\text { la demande d'importation }\end{array}$ \\
\hline
\end{tabular}

domestique, d'où la recherche d'un substitut sous la forme de sucre et d'éthanol en provenance de Brésil. Le riz parce que l'envolée de son cours international, cette fois en fin de période de hausse des prix (i.e., sur la campagne 2007/08), serait surtout liée à l'adoption de mesures politiques par de nombreux états, acteurs importants sur le marché du riz, visant à privilégier le marché domestique; dans le contexte d'un marché mondial du riz très étroit, ces mesures auraient amplifié le mouvement à la hausse du prix international du riz. II serait néanmoins imprudent de résumer les histoires des cours internationaux du sucre et du riz à ces seules explications spécifiques. Dit autrement, ces explications spécifiques doivent être replacées dans le contexte général des déterminants qui ont conduit au mouvement de hausse de nombreuses matières premières agricoles, i.e., dans le contexte du mouvement tendanciel à l'œuvre depuis le début des années 1990 (atonie de l'offre et dynamisme de la demande alimentaire, pour l'alimentation humaine et celle des animaux), mouvement possible de porter un jugement définitif sur le rôle de la spéculation.

Dans ce contexte général que l'on peut résumer ainsi : la hausse des cours agricoles qui s'est étendue de l'été 2006 à la fin du printemps 2008 serait due aux tendances de l'offre et de la demande à l'œuvre depuis environ deux décennies, tendances amplifiées par plusieurs évolutions favorables à la hausse depuis les années 2000 et des événements conjoncturels lors des années 2006, 2007 et 2008, il serait intéressant $d$ 'analyser en profondeur l'impact de la variable stocks. Plus spécifiquement, il conviendrait de tester la validité de l'hypothèse selon laquelle les stocks auraient atteint, à compter du milieu de l'année 2006, des niveaux "trop bas", inférieurs aux niveaux minima retenus par la FAO [25] pour jouer un quelconque rôle de stabilisation des prix. Mieux encore, dans une conjoncture d'offre rigide à court terme (2006 et 2007) et de demande inélastique (celle pour I'alimentation humaine comme celle destinée à la fabrication de biocarburants, mandats d'incorporation obligent), l'effet d'une diminution additionnelle des stocks aurait un effet plus que proportionnel sur les prix agricoles ${ }^{28}$.

Malheureusement, et c'est le deuxième enseignement général de notre analyse, force est de constater que les travaux réalisés à ce jour ne permettent pas de quantifier, sans ambiguïté, la responsabilité de tel ou tel déterminant au mouvement de hausse, puis de baisse, des prix agricoles (même quand cette responsabilité est non contestée, a fortiori quand elle est l'objet de controverses). Comme nous avons déjà eu l'occasion de le souligner en introduction, l'imprécision provient pour partie du caractère « non scientifique » des analyses qui se présentent (très) souvent sous la forme de rapports non validés via des publications en appui dans des revues à comité de lecture, voire sous la forme d'études réalisées par des organisations et même des institutions dont on peut légitiment douter de la neutralité. Pour partie, cela vient également du fait que ce mouvement de hausse puis de baisse des prix agricoles est (très) récent; en outre, comme le notent Keyzer et al. [6], les statistiques disponibles sont insuffisamment détaillées pour rendre compte, par exemple, de la réelle importance de la spéculation sur les mar-

\footnotetext{
${ }^{28}$ Sur ce point, voir Keyzer et al. [6] qui, de façon générale, soutiennent l'hypothèse qui ferait jouer aux stocks (à leurs bas niveaux) un rôle clef dans la hausse des cours. Headey et Fan [24] se posent la même question, dans une perspective néanmoins plus critique dans la mesure où l'évolution des stocks est pour partie endogène (i.e., résulte des évolutions des offres et de demandes), pour partie exogène (i.e., fruit de décisions privées et publiques pour qu'il en soit ainsi).
} 
chés agricoles à compter de l'été 2007 et sur la première moitié de l'année 2008, puis du reflux de cette spéculation à compter de la deuxième partie de l'année 2008 (cf. supra). On peut espérer que le temps aidera, au moins pour partie, à surmonter ce handicap de l'insuffisant recul face à l'événement (les premières études dans des revues scientifiques à comité de lecture commencent à apparaître ; cf., par exemple, le numéro spécial de la revue Agricultural Economics paru à la fin de l'année 2008). II ne faudrait pas tirer de ce deuxième enseignement une conclusion exagérément pessimiste, à savoir l'inutilité de l'analyse économique et des modèles quantitatifs des marchés agricoles. Bien au contraire! L'analyse économique est plus que jamais nécessaire pour au moins deux raisons : la première dans une perspective, celle que nous avons essayée de suivre dans ce papier, d'explicitation et de compréhension des mécanismes de formation des prix agricoles et de leurs évolutions; la deuxième, conséquence logique et indispensable de la première, dans l'objectif de construire des outils quantitatifs pertinents, i.e., capturant les différents mécanismes via lesquels les facteurs explicatifs potentiels des prix agricoles et de leurs évolutions agissent. À cet égard, les modèles agricoles internationaux (et nationaux) aujourd'hui à disposition sont défaillants sur plusieurs points : plusieurs de ces modèles ont en effet été élaborés dans une optique essentiellement tournée vers l'analyse des conséquences de réformes des politiques agricoles dans le cadre des négociations agricoles multilatérales, hier du cycle de I'Uruguay, aujourd'hui du cycle de Doha; quant aux modèles utilisés pour bâtir des perspectives, leur horizon d'analyse est le moyen terme, d'où une inadaptation à une utilisation pour l'étude d'un mouvement de hausse puis de baisse sur courte période. En outre, les deux types de modélisation souffrent d'un certain nombre $d^{\prime}$ insuffisances auxquelles il convient de remédier.

Le mouvement de hausse puis de baisse des prix agricoles que nous venons de connaître appelle en effet, de façon urgente et impérieuse, à un enrichissement des modèles quantitatifs, explicatifs et prospectifs, d'analyse des marchés agricoles. Plus que d'expliquer l'épisode de hausse des prix agricoles, il s'agit avant tout de se doter d'outils à même de rendre compte des fluctuations des cours agricoles. Dans ce contexte, deux pistes d'amélioration des modèles existants doivent être privilégiées : d'une part, la prise en compte des risques (de prix et de quantités) et de leurs conséquences sur les comportements des acteurs, les agriculteurs en premier lieu, mais aussi les organismes de collecte et de stockage, les transformateurs, les exportateurs et les importateurs, les distributeurs, les spéculateurs, etc. ; et, d'autre part et de façon étroi- tement liée, la compréhension des formations des anticipations de prix qui dictent les décisions en termes d'allocation des surfaces, de rendements visés, de quantités stockées/déstockées, etc. Par ailleurs et de façon tout aussi importante, les modèles agricoles existants doivent être enrichis par l'ajout explicite de modules permettant de rendre compte des interactions croissantes entre, d'une part, les marchés agricoles et ceux de l'énergie (aujourd'hui celui du pétrole, demain sans doute ceux d'autres énergies), et, d'autre part, les marchés agricoles et les marchés financiers.

\section{RÉFÉRENCES}

1. FAO. La flambée des prix des denrées alimentaires: faits, perspectives, effets et actions requises. Papier préparé pour la Conférence de haut niveau sur la sécurité alimentaire mondiale « les défis du changement climatique et des bioénergies », Rome, 3-5 juin 2008.

2. Gohin A. Les raisons de l'augmentation des prix agricoles: une revue de littérature. Miméo UMR SMART. Rennes: INRA et AgroCampus Ouest, 2008.

3. Mitchell M.

4. Berthelot J. Les causes de l'essor et de l'éclatement de la bulle des prix agricoles. Papier présenté au colloque de la SFER du 06 novembre 2008, La crise des matières premières agricoles: le regard des chercheurs, Paris. 2008.

5. IFPRI. Livestock to 2020: The Next Food Revolution. IFPRI 2020 Brief $n^{\circ}$ 61, Washington DC, 1999.

6. Keyzer $M$, Merbis $M$, Nubé $M$, van Wesenbeeck L. Food, Feed and Fuel: When Competition Starts to Bite. Amsterdam : Centre for World Food Studies (SOW-VU), 2008.

7. Banse M, Nowicki P, van Meijl H. Why are Current World Food Prices so High? LElWageningen UR, Report 2008-040, 2008.

8. Trostle R. Global Agricultural Supply and Demand: Factors Contributing to the Recent Increase in Food Commodity Prices. USDA, ERS, WRS-0801, May 2008 (Revised July 2008).

9. Séronie JM. Marchés agricoles : à pile ou face ? CER France, Veille économique agriculture, octobre, page $1: 2008$.

10. Mollière $C$. Le point sur les matières premières végétales. Crédit Agricoles. Eclairages 2008 ; 128 : 15-6 (décembre).

11. Bergougnoux J, Guyomard H. Diagnostic stratégique France 2025, Groupe 6, Ressources rares et environnement : rechercher les voies d'un développement durable dans un monde incertain. Paris : Conseil d'Analyse Stratégique (CAS), 2008.

12. European Commission. High Prices on Agricultural Commodity Markets: Situation and Prospects; A Review of Causes of High Prices and
Outlook for World Agricultural Markets. EC, DG for Agriculture and Rural Development, Directorate L, July 2008.

13. Von Braun J. The World Food Situation: New Driving Forces and Required Actions. IFPRI's Biannual Overview of the World Food Situation presented to the CGIAR Annual General Meeting, Beijing, December 4, 2007.

14. European Commission. Prospects for Agricultural Markets 2000-2007. EC, DG for Agriculture and Rural Development, November 2000.

15. European Commission. Prospects for Agricultural Markets 2001-2008. EC, DG for Agriculture and Rural Development, July 2001.

16. FAO. Growing Demand on Agriculture and Rising Prices of Commodities: An Opportunity for Smallholders in Low-Income, AgriculturalBased Countries? Paper prepared for the Round Table Organized during the thirty-first session of IFAD's Governing Council, 14 February 2008.

17. Klatzmann J. Attention statistiques! Comment déjouer les pièges. Paris: Editions La Découverte, 1996.

18. Bachelier B. Les biocarburants ne menacent pas la sécurité alimentaire. Propos cités dans OCDE, 2008, Notes du CSAO (Club du Sahel et de I'Afrique de l'Ouest); Extrait de la publication "l'Afrique de l'Ouest peut-elle se nourrir?" 2008.

19. Courleux F. De la hausse à la baisse des prix, impacts de la crise économique sur l'agriculture et les IAA : éléments de contexte et propositions pour anticiper l'avenir. Document de travail du MAP, Sous-direction de la prospective et de l'évaluation, Bureau de la prospective, 2008.

20. OCDE. Impact relatif sur les prix mondiaux des produits des changements structurels à court et long terme sur les marchés agricoles; le choc des prix des matières premières: le retournement structurel d'une tendance de prix bas ? TAD/CA/APM/CFS/MD(2008)3. 2008.

21. Abbott $P$, Hurt $C$, Tyner WE. What's Driving Food Prices? Ferm Foundation Issue Report, July 2008.

22. Collins K. The Role of Biofuels and Other Factors in Increasing Farm and Food Prices: A Review of Recent Developments with a Focus on Feed Grain Markets and Market Prospects. Report written as supporting material for a review conducted by Kraft Foods Global, Inc. of the current situation in farm and food markets, June 19, 2008.

23. CBC. Is Food Commodity Securization Worsening the World's Food Problem? Policy Brief, Conference Board of Canada, 2008.

24. Headey D, Fan S. Anatomy of a Crisis: The Causes and Consequences of Surging Food Prices. Agricultural Economics $2008 ; 39$ : 375-81.

25. FAO. Approaches to World Food Security. Economic and Social Development Paper 2003; $\mathrm{n}^{\circ}$ 32, Rome. 